\section{Utilization Behavior}

Catherine C. Price ${ }^{1}$ and David J. Libon ${ }^{2}$

${ }^{1}$ Department of Clinician and Health Psychology \& Dept of Anesthesiology, University of Florida, Florida, USA

${ }^{2}$ Departments of Geriatrics, Gerontology, and Psychology, Rowan University, New Jersey Institute for Successful Aging, School of Osteopathic Medicine, Stratford, NJ, USA

\section{Synonyms}

Imitation behavior

\section{Definition}

Utilization behavior is the act of grasping or using objects that are within reach or in the field of vision. While objects may be used correctly, the behavior occurs in a context that is inappropriate.
For example, when shown a pair of glasses, a patient is likely to put on the glasses. The behavior occurs with unilateral, or bilateral, frontal lesions, particularly orbitofrontal lesions (Lhermitte 1983). Classically, utilization behavior is attributed to an imbalance between frontal and parietal lobes, such that the frontal lobes can no longer inhibit the parietal lobe's dependency upon sensory or environmental input. Utilization behavior is one type of environmental dependency, but not to be confused with magnetic apraxia.

\section{Cross-References}

Environmental Dependency

- Magnetic Apraxia

\section{References and Readings}

Lhermitte, F. (1983). 'Utilization behavior' and its relation to lesions of the frontal lobes. Brain, 106, 237-255. 